\section{VELCADE Bortezomib}

\section{Multiples Myelom: Steigerung der Wirksamkeit von Velcade ${ }^{\circledR}$ (Bortezo- mib) durch Kombinationstherapie}

Die APEX-Studie zeigt: Velcade ${ }^{\circledR}$ (Bortezomib) erreicht im Vergleich zu einer Therapie mit hoch dosiertem Dexamethason einen signifikanten Überlebensvorteil und ist die effektivste Einzelsubstanz bei Patienten mit nur einer Vortherapie. Der innovative Wirkstoff erzielt in diesem Stadium der Erkrankung eine Ansprechrate von $45 \%$. Bislang kann Bortezomib deshalb als Monotherapie für Myelom-Patienten ab dem ersten Rezidiv eingesetzt werden, die bereits eine Stammzelltransplantation erhalten haben oder sich für diese nicht eignen. Studien bei Patienten mit rezidiviertem oder refraktärem multiplem Myelom belegen darüber hinaus, dass die Kombination des Proteasom-Inhibitors mit Dexamethason im Rahmen der Standardbehandlung das Ansprechen im Vergleich zur Monotherapie deutlich verbessern kann. Außerhalb der zugelassenen Indikation untersuchen deshalb mehrere weiterführende Studien den Einsatz von Bortezomib zusammen mit anderen Wirkstoffen.

Was passiert, wenn man eine erfolgreiche Monosubstanz wie Bortezomib mit anderen Wirkstoffen kombiniert? Eine Ant- wort auf diese Frage gibt eine Untersuchung von Jagannath et al. In dieser Phase-II-Studie erhielten Patienten mit rezidiviertem oder refraktärem multiplem Myelom und mindestens einer Vortherapie bei fortschreitender Erkrankung nach 2 Zyklen oder stabiler Erkrankung nach 4 Zyklen zusätzlich zu Bortezomib $20 \mathrm{mg}$ orales Dexamethason. Das Resultat: Die Hinzunahme von Dexamethason führte zu einer gesteigerten Response. So betrug das Ansprechen $(\mathrm{CR}+\mathrm{PR})$ auf Bortezomib $\left(1,3 \mathrm{mg} / \mathrm{m}^{2}\right.$ $\mathrm{KOF}$ ) $38 \%$ in der Monotherapie gegenüber $50 \%$ in der Kombination. Insgesamt hatten dabei 28 Patienten (52\%) zusätzlich Dexamethason erhalten.

Mit diesen Ergebnissen bestätigten sich auch die Resultate der Zulassungsstudie SUMMIT. Dort hatten 74 von 202 stark vorbehandelten Patienten mit refraktärem multiplem Myelom bei mangelndem Ansprechen zusätzlich Dexamethason erhalten, bei $18 \%$ von ihnen konnte ein verbessertes Ansprechen festgestellt werden. Das Nebenwirkungsprofil war in beiden Studien vorhersehbar und gut beherrschbar.

\section{Viel versprechende Ergebnisse}

weiterführender Studien

Darüber hinaus wird außerhalb der aktuellen Indikation der Einsatz von Bortezomib in Kombination mit anderen Wirkstoffen überprüft. Die Forscher untersuchen dabei unter anderem den Nutzen des Wirkstoffs im Rahmen einer Kombination als Primärtherapie vor Stammzelltransplantation. Hier ist ne- ben einer sehr guten Wirksamkeit insbesondere wichtig, dass die Behandlung keinen negativen Einfluss auf die Stammzellen hat - erste Resultate sind bisher viel versprechend.

Beispielsweise konnten Oakervee et al. im Rahmen einer Dosiseskalationsstudie belegen, dass eine Induktions-Therapie aus Bortezomib, Doxorubin und Dexamethason zu einem sehr hohen Ansprechen führt und eine anschließende Sammlung von Stammzellen nicht einschränkt : So sprachen 20 der 21 Patienten $(95 \%)$ bei moderaten Nebenwirkungen auf die Behandlung an (CR + PR). $5(24 \%)$ von ihnen kamen sogar in eine komplette Remission. Autologe Blutstammzellen konnten ebenfalls bei 20 von 21 Patienten mobilisiert werden, eine anschließende Therapie mit hoch dosiertem Melphalan sowie eine Stammzelltransplantation erhielten bislang 18 Patienten. Ingesamt machen die Ergebnisse deutlich, dass das Potenzial von Bortezomib noch lange nicht erschöpft ist. Unter anderem wurde für die Primärtherapie bereits eine Zulassungsstudie initiiert, und auch bei weiteren Tumorentitäten wird die Wirksamkeit des Proteasom-Inhibitors erforscht.

\section{Referenzen:}

Richardson P. et al.: N Engl J Med. 2005; 352:2487-2498. Richardson P. et al.: N Engl J Med. 2003;348:2609-2617. Oakervee H. et al.: Br J Haematology 2005;129:755-762.

Weitere Informationen bei

Kerstin Aschoff

Janssen-Cilag GmbH

Tel. +49 2137 955-412

kaschoff@jacde.jnj.com

\title{
PharmaTicker+++ PharmaTicker+++ PharmaTicker+++ PharmaTicker+++
}

Pfrimmer Nutricia GmbH. Die klinische Bedeutung von Mangelernährung wird noch immer unterschätzt. Dies ist ein Ergebnis einer repräsentativen Umfrage bei 200 niedergelassenen Allgemeinärzten, Praktikern und Internisten durch EMNID, die im August 2005 im Auftrag von Pfrimmer Nutricia durchgeführt wurde. Weitere Informationen bei

Burson-Marsteller GmbH \& Co.KG, Telse Friccius

Tel.: + 4969 23809-45, Fax -71

Telse_Friccius@de.bm.com
Olympus Deutschland GmbH. Mit zwei Koloskopen und zwei Gastroskopen sind nun die ersten Geräte der neuen Endoskopgeneration EXERA II verfügbar. Mit der einzigartigen Bildqualität und der besonderen Ausstattung der neuen Produkte festigt Olympus seine Technologie. und Innovationsführerschaft im Bereich der medizinischen Endoskopie.

Weiter Informationen bei

Dorothea Küsters Life Science Communications $\mathrm{GmbH}$

Dr. Michael Hubert

Tel. +49 69 61988-16, Fax -10

hubert@dkcommunications.de
MediGene AG. Das deutsch-amerikanische Biotechnologie-Unternehmen hat den Beginn einer klinischen Phase-II-Studie mit dem Medikamentenkandidaten EndoTAG ${ }^{\circledR}$-1 zur Behandlung des fortgeschrittenen Pankreaskarzinoms bekannt gegeben. Eine Interimsanalyse mit den Daten der ersten 100 Patienten ist für Ende 2006 geplant.

Weitere Informationen bei

MediGene AG, Dr. Georg Dönges

Tel. +49 89 8565-3317, Fax -2920

investor@medigene.com 


\section{MA}

\section{sanofi aventis}

Das Wichtigste ist die Gesundheit

\section{TAX 311 schenkt Zuversicht beim metastasierten Mammakarzinom}

Taxotere ${ }^{\circledR}$ (Docetaxel) zeigt im direkten Vergleich mit Paclitaxel ein erheblich verlängertes Gesamtüberleben und eine deutlich verlängerte Zeit bis zur Tumorprogression - so die Ergebnisse der TAX 311 Studie, die das Journal of Clinical Oncology in seiner letzten Ausgabe veröffentlicht hat. In der Studie wurden Patientinnen mit metastasiertem Mammakarzinom behandelt, die sich zuvor einer Anthrazyklin-basierten Therapie unterzogen hatten. Das Gesamtüberleben konnte gegenüber Paclitaxel mit 12,7
Monaten auf 15,4 Monate gesteigert werden.

An der randomisierten Phase-III-Studie nahmen 449 Frauen mit lokal fortgeschrittenem oder metastasiertem Brustkrebs teil. Ihnen wurde entweder Taxotere $100 \mathrm{mg} / \mathrm{m}^{2}$ (1-h-Infusion, alle 21 Tage) oder Paclitaxel 175 mg/m² (3-hInfusion, alle 21 Tage) verabreicht. Der Vergleich der beiden Taxane ist in seiner Form bisher einzigartig und bestätigt die vermuteten Unterschiede in der Wirksamkeit von Taxotere und Paclitaxel. "Die Ergebnisse dieses Head-to-HeadVergleichs liefern Onkologen den medizinischen Beweis für diese Unterschiede. Selten haben wir in Studien derart signifikante Unterschiede gesehen, die sich auf das Gesamtüberleben auswirken.”, so Dr. Stephen E. Jones, medizinischer Leiter des US Oncology Research und Leiter der Abteilung für Brustkrebs-
Forschung des Baylor-Sammons Cancer Centers in Dallas, Texas.

Die Intent-to-treat Analyse zeigt eine Verlängerung des Gesamtüberlebens $(15,4$ vs. 12,7 Mo. $p=0,03)$ und des progressionsfreien Überlebens mit Taxotere gegenüber Paclitaxel (5,7 vs. 3,6 Mo. p < $0,0001)$. Das Gesamtansprechen ist mit 32 vs. $25 \%$ bei Taxotere höher ( $p=0.10$ n.s.). Die Ansprechzeit war bei den mit Taxotere behandelten Patientinnen deutlich länger (7,5 Mo. vs. 4,6 Mo. p = 0,01). In der Analyse der für das Ansprechen auswertbaren Patientinnen zeigte sich ein deutlicher Unterschied in der Ansprechrate (37 vs. $26 \%, \mathrm{p}=0,02$ ).

Weitere Informationen bei

Sanofi-Aventis Deutschland $\mathrm{GmbH}$

Cristina Iannazzo

Industriepark Höchst, Gebäude F821

65926 Frankfurt a. M., Deutschland

Cristina.Iannazzo@sanofi-aventis.com

\section{Merck beantragt Indikationserwei- terung für Erbitux ${ }^{\circledR}$ zur Behandlung von Kopf- und Halskarzinomen}

Merck hat bei der Europäischen Arzneimittel-Agentur (EMEA) und der SwissMedic die Erweiterung der Indikationen für das spezifische Tumorpräparat Erbitux $^{\circledR}$ (Cetuximab) zur Behandlung von Plattenepithelkarzinomen des Kopfes und Halses beantragt. Die beantragte neue Indikation sieht die Anwendung von Erbitux in Kombination mit einer Strahlentherapie für lokal fortgeschrittene Plattenepithelkarzinome des Kopfes und Halses und als Monotherapie bei Patienten mit Rezidiv und/oder Metastasen vor, bei denen eine vorherige Chemotherapie nicht angeschlagen hat. Mit der Zulassung für die Behandlung von Plattenepithelkarzinomen des Kopfes und Halses könnte Erbitux eine neue, wirksame und gut verträgliche Therapieoption für diese schwierige und immer häufiger auftretende Krebsart werden. Erbitux ist ein monoklonaler Antikörper, der den epidermalen Wachstumsfaktorrezeptor (EGFR) blockiert, der für das Tumorwachstum verantwortlich ist und bei einer Reihe von verschiede- nen Krebserkrankungen eine Rolle spielt. «Kopf- und Halskarzinome stellen eine echte Herausforderung für die Medizin dar», so Dr. Bernhard Ehmer, Leiter der Geschäftseinheit Onkologie bei Merck. «Nur etwa 33\% der Patienten sind 5 Jahre nach der Diagnose noch am Leben, und leider gibt es immer noch zu wenige Behandlungsoptionen. Erbitux hat deutliche Vorteile bei Patienten mit diesen Erkrankungen gezeigt.»

Weitere Informationen bei

IntraMedic $\mathrm{GmbH}$

Dr. Cornelia Bartels

Tel. +49 61027993 338, Fax -301

\section{PharmaTicker+++ PharmaTicker+++ PharmaTicker+++ PharmaTicker+++}

Hoffmann-La Roche AG. Für Frauen mit rezidiviertem oder metastasiertem Brustkrebs liegt jetzt ein neuer Informationsfilm mit Begleitbroschüre auf DVD vor. «Wenn Brustkrebs wiederkommt» kann kostenlos bestellt werden unter dem Stichwort "Durch die Brust ins Herz", Postfach 5111 70, 50947 Köln oder über www.brustkrebszentrale.de

Weitere Informationen bei

art tempi communications gmbh, Heike Balogh

Tel. $+49221272359-70$

balogh@art-tempi.de
Novartis Pharma GmbH. Die amerikanische Food and Drug Administration (FDA) hat einer vorrangigen Prüfung von Femara ${ }^{\circledR}$ (Letrozol) bei der adjuvanten Behandlung postmenopausaler Frauen mit Hormonrezeptor-positivem Brustkrebs im Frühstadium zugestimmt. Der Zulassungsantrag für die Anwendung von Femara im adjuvanten Bereich wurde im Juni 2005 weltweit eingereicht.

Weitere Informationen bei

Novartis Pharma GmbH, Dr. Michaela Paudler-Debus

Tel. +49 911 273-12462, Fax -12971

michaela.paudler-debus@novartis.com
Lilly Pharma GmbH. Bei der World Conference on Lung Cancer (WCLC) wurden aktuelle Daten präsentiert, die zeigen, dass eine Behandlung mit Alimta (Pemetrexed) in Kombination mit Cisplatin Patienten mit malignem Pleuramesotheliom größere Überlebensvorteile bringt, als bisher berichtet.

Weitere Informationen bei

3K Agentur für Kommunikation $\mathrm{GmbH}$

Sabine Theobald

Tel. +49 69 971711-0, Fax -22

info@3K-Komm.de

\begin{tabular}{ll}
\hline KARGER & ๑ 2005 S. Karger GmbH, Freiburg \\
Fax +4976145207144 & $\begin{array}{l}\text { Accessible online at: } \\
\text { www.karger.com/onk }\end{array}$
\end{tabular}

